Mundo Agrario vol. 18, n 37, e044, abril 2017. ISSN 1515-5994

Universidad Nacional de La Plata.

Facultad de Humanidades y Ciencias de la Educación.

Centro de Historia Argentina y Americana

\title{
Territorios encadenados, tránsitos migratorios y ruralidades adaptativas
}

\author{
Enchained territories, migratory displacements and adaptive \\ ruralities
}

Luis Camarero*

* Universidad Nacional de Educación a Distancia (UNED), España | Icamarero@poli.uned.es

\section{PALABRAS CLAVE}

Regímenes agroalimentarios

Mercados laborales rurales

Movilidad

Ruralidad

España

\section{KEYWORDS}

Agri-food regimes

Rural labour markets

Mobilities

Spain

\section{RESUMEN}

El texto se interesa por las formas en que los territorios, en este caso las distintas áreas y localidades rurales, se integran, vinculan o desvinculan en procesos y cadenas productivas y económicas de orden global. La conexión entre territorios y cadenas económicas se produce mediante flujos de mercancías, insumos y capitales, pero también mediante movimientos migratorios y prácticas diversas de movilidad. La movilidad en su sentido amplio determina la relación y posicionamiento de trabajadores y consumidores en el espacio del territorio y en la secuencia de las cadenas globales. El proceso de división social del trabajo genera nuevas lógicas de integración/desintegración de las regiones en el proceso socioeconómico, y diferentes demandas de movilidad asociadas a dichos cambios. Los territorios se adaptan y se reorganizan para insertarse en distintos momentos de las cadenas de producción de valor. Los lugares y territorios llegarán -y esta es la hipótesis que encierra este texto- a insertarse en cadenas globales en la medida en que desarrollen capacidades de adaptabilidad a las condiciones productivas y especialmente consigan reducir la fricción territorial garantizando la gestión migratoria y movilidad de la mano de obra. Con este propósito se contempla la evolución socioagraria de las áreas rurales en España desde finales del siglo XIX.

\section{ABSTRACT}

The text is concerned with the ways in which the territories, in this case the different rural areas and localities, are integrated, linked or dissociated into processes and chains of production and economic of a global order. The connection between territories and economic chains occurs through flows of goods, inputs and capital, but also through migratory movements and diverse mobility practices. The process of social division of labor generates new logics of integration / disintegration of the regions in the socioeconomic process, and different mobility demands associated with these changes. The hypothesis that encloses this text is that places and territories will reach to insert in global chains if they develop capacities of adaptability to the productive conditions and especially they manage to reduce the territorial friction guaranteeing the migratory management and mobility of the labor force. With this point of view the socio-agricultural evolution of the rural areas in Spain is contemplated from the end of century XIX 


\section{Introducción}

En líneas generales, durante el siglo XX ha culminado el proceso de desaparición de las unidades de producción doméstica agraria, basadas en formas de vida cotidiana muy dependientes del entorno de los pueblos y aldeas que caracterizaban el paisaje social de las áreas rurales del sur de Europa. De forma paralela al ocaso de las economías campesinas y agrarias ha avanzado el proceso de globalización económica. Este proceso ha sido especialmente patente en el desarrollo del comercio internacional primero, en la estandarización del consumo después, y en la extensión de las cadenas de valor agroalimentarias finalmente.

Una vez entrado el siglo XXI las áreas rurales del suroeste de Europa conforman un archipiélago de situaciones territoriales dispersas que mezclan formas muy distintas de integración en el sistema económico global. Como ha puesto en evidencia Veltz (1996), aunque los procesos globales afectan por igual a los territorios, las respuestas que ofrecen cada una de las localidades resultan muy distinta. Por esta razón encontramos áreas vecinas pero muy diferentes en sus formas de vinculación a los procesos globales de generación de valor. Así, al lado de zonas del interior de Europa, caracterizadas por un despoblamiento secular agónico, encontramos lugares que albergan actividades de innovación socioeconómica con orientaciones muy diferentes. Pueden destacarse, por un lado, las economías simbióticas en las áreas del litoral mediterráneo conformadas a través de modelos muy flexibles de integración de turismo y agricultura industrial; $\mathrm{y}$, por otro lado, encontramos lugares que aprovechan las oportunidades de movilidad y conectividad espacial y conforman localidades híbridas urbano-rurales.

Desde la constatación de la diversidad de respuestas frente a idénticos procesos, el artículo quiere situar la evolución reciente de las áreas rurales del sur de Europa en términos de adaptación a procesos de orden global. El papel que han tenido las economías agrarias rurales en el contexto de la mundialización primero y de la globalización después ha sido bien estudiado (Friedman \& McMichael, 1989; Etxezarreta, 2006). Sin embargo, el papel intrínseco que tienen las áreas rurales en el proceso de organización de cadenas de mano de obra globales del sistema-mundo ha sido menos explorado. La tesis de la reestructuración rural (Lowe, Marsden \& Munton, 1990) ha mostrado que las áreas rurales no son sólo lugares destinados a la producción de alimentos o materias primas. El papel de éstas es creciente en la regulación de los flujos demográficos (Litcher \& Brown 2011). Y además en el contexto de las economías postproductivas (Wilson, 2001) se ha venido destacando la importancia que tienen las identidades locales en la atribución de valor de las producciones. Ambas cuestiones, movilidad y producción de significados, deben considerarse para comprender la posición que ocupan las pequeñas regiones en el orden territorial global.

El marco propuesto por Wallerstein (1974) de sistema-mundo para explicar el proceso de articulación global de un sistema económico resulta muy sugerente para interpretar el proceso de integración de las economías rurales en los sistemas socioeconómicos nacionales. El planteamiento que hacen Lash y Urry (1994) de las economías del signo-espacio ofrece un marco analítico en el que integrar de forma conjunta el desarrollo de los procesos de intercambio de mercancías, población y signos en la conformación de sistemas de orden transnacional. Desde la óptica territorial resulta especialmente relevante la noción de desarticulación. Bair y Werner (2011) extienden la noción de integración de territorios a la dinámica completa de enganche y desenganche. Es decir, se ocupan no sólo del éxito sino también del posterior abandono y salida de las actividades económicas. De esta forma, es posible interesarse por el proceso adaptativo que experimentan las áreas rurales interpretadas como ciclos en su conexión/desconexión de los procesos y cadenas productivas globales. Desde la reestructuración rural (Massey, 1984; Lowe, Marsden \& Munton, 1990) ha mostrado el impacto de las olas de inversión y desinversión como estrategias de acumulación asociadas a la explotación de desigualdades territoriales.

En este texto, en primer lugar describiremos brevemente distintas fases que marcan la evolución de las formas de funcionamiento del agro en cuanto formas socioproductivas, es decir, en el análisis se incluyen y 
enfatizan las relaciones de trabajo en las que se sustentan. En segundo lugar, contemplaremos la evolución de las áreas agrarias de España dentro del marco de la pertenencia al sistema-mundo y de su papel dentro del funcionamiento de los regímenes agroalimentarios.

Como han señalado Moran, Blunden, Workman y Bradly (1996), la observación de las formas reales de regulación sólo puede realizarse desde el ámbito nacional. Por ello, la escala de análisis utilizada es la de un estudio regional centrado en el conjunto de España. Sin embargo, parece posible extrapolar los principales resultados a otros lugares del sur de Europa que mantienen condiciones estructurales y productivas similares.

Cuando el propósito es la observación de procesos adaptativos, el ámbito de observación temporal es necesariamente largo. Por ello, para comprender el periodo de la globalización, tenemos que remitirnos, como estrategia comparativa para visualizar cambios en las tendencias, al periodo de la mundialización (Castells, 1997). Con esta intención se incluyen datos desde finales del XIX -momento álgido de la mundialización económica y consolidación de la división regional del trabajo (Wallerstein, 1974) a través de la expansión del sistema colonial-.

\section{Sistemas regionales de división social del trabajo y regímenes agroalimentarios}

Wallerstein (1974) caracteriza al sistema mundial por la división extensiva del trabajo según lógicas geográficas. Es una división territorial del trabajo que no responde necesariamente a condiciones ecológicas o ambientales, sino que es resultado de la extensión de la división funcional u ocupacional sobre el territorio. Tiene su fundamento en el propio sistema de división social del trabajo que permite la acumulación y apropiación y añade el territorio como sistema de jerarquización y elemento de dominación social. (Delgado, Carpintero, Lomas \& Sastre, 2014). Buena parte de la historia de la conformación del sistema socioeconómico mundial viene determinada por la asignación de grandes regiones rurales como productoras y por la concentración de la transformación y del consumo final en áreas urbanas localizadas continentalmente. Dicha distinción sanciona en buena medida la diferenciación entre las regiones-países centrales y las regiones-países periféricos.

Friedman y McMichael (1989) avanzaron en la noción de régimen alimentario que ha generado un marco ampliamente aceptado para interpretar el devenir de las transformaciones en el ámbito agrario. Un régimen agroalimentario es un conjunto de reglas e instituciones que regulan la producción agraria y alimentaria dentro de un sistema de acumulación de capital. Inicialmente se hablaba de dos regímenes, pero el debate e investigación desde distintas ópticas ha propiciado la distinción, desde los años 80, de otros momentos diferenciados del segundo. McMichael (2005) especifica la aparición del denominado régimen corporativo alimentario, mientras que otros autores como Pistorius y Van Wijk (1999) llegan a diferenciar un tercer régimen a partir del desarrollo biotecnológico.

El primer régimen tiene lugar, según Friedman y McMichael (1989), en el periodo que va desde la revolución industrial hasta la primera guerra mundial. Este régimen se caracteriza por la creciente división regional en la producción de alimentos y manufacturas, y por el desarrollo de un mercado internacional soportado por el librecambio que es profundamente desigual entre regiones manufactureras y las agrarias. La agricultura se consolida como una producción a escala mundial y se establecen diferencias entre lugares productores y consumidores. El fuerte liberalismo imperante pone en juego y estimula la competencia de un sistema de mercados transnacionales a gran escala.

El segundo periodo adquiere forma plena después de la segunda guerra mundial. Este periodo coincide con el tránsito metafórico de "la cocina a la factoría" (Pilcher, 2006) que refiere el cambio en las formas de alimentación. Se reduce el consumo de productos frescos para favorecer el de productos procesados. La 
producción agraria se convierte principalmente en input de procesos de elaboración de alimentos u otros bienes. El empeño del sistema agropecuario está en producir materias primas que integren el proceso de transformación antes que productos de consumo directo. Esta tendencia es extrapolable a toda la producción agropecuaria, incluso cuando su destino no sea la alimentación humana. Muestra de ello es el crecimiento de la ganadería alimentada con piensos y del peso que su aporte tiene en el conjunto alimentario final. Para dirigir el proceso de transformación y cambio resulta crucial que el estado tenga un papel de fuerte intervención, por ejemplo mediante la subvención y promoción de nuevas variedades industriales. En este segundo periodo la producción de valor se desplaza desde el propio proceso de producción hasta el proceso de transformación.

Distintos autores diferencian, desde finales de los años 80, un tercer régimen de acumulación alimentaria. Este grupo de autores coinciden en destacar la erosión de la regulación estatal y el poder directivo de los mercados. Dos elementos darían soporte al tercer régimen. Por una parte, el crecimiento de la biotecnología, que ha desplazado la acumulación de valor a otros segmentos económicos (farmacéuticas, laboratorios y especialmente empresas energéticas) y, por otra parte, el valor que como mercancía de cambio adquieren ciertos productos agrarios, hecho que les ha permitido establecerse como productos que soportan valores financieros.

En este sentido Levidow (2015) apunta la transición del régimen corporativo alimentario hacia lo que viene a denominarse corporativo-ambiental. El peso de los biocombustibles y la intensificación sostenible generan un nuevo contexto en el que se desarrolla la "acumulación verde". Levidow en su análisis observa la continuidad de las prácticas neoliberales de regulación y organización de las producciones, y de los mercados y la supremacía de éstas sobre las lógicas ambientalistas. Por su parte, Pechlaner y Otero (2008) consideran un tercer régimen caracterizado por el crecimiento de la agricultura biotecnológica y por la creciente liberalización del comercio agrario y alimentario. Observan que existe una interacción tan fuerte entre desarrollo biotecnológico y liberalismo que llegan a señalar que "el tercer régimen alimentario podría acertadamente denominarse 'régimen neoliberal alimentario'” (p. 366). Al control de las reglas y el uso de las nuevas tecnologías se une la financiarización del sistema alimentario como estrategia de expansión corporativa (Delgado, 2010).

Van der Ploeg (2016) ha resumido estas transformaciones señalando la desconexión progresiva de las economías agroalimentarias respecto al entorno. Por una parte, las producciones alimentarias se desconectan de los recursos naturales por el desarrollo de condiciones de crecimiento, transporte y almacenamiento artificial. Por otra parte, la actividad agraria se hace cada vez más independiente de las capacidades, conocimientos y expectativas de los actores que intervienen en la medida en que los procesos productivos se van segmentando y secuenciando hasta convertirse en unidades independientes e indiferenciadas. Por último, las economías agroalimentarias se desconectan de la sociedad en general mediante la puesta en marcha de sistemas globales de distribución que dificultan la conexión de los consumidores con el territorio.

Visto desde el territorio, con estas condiciones se generan nuevas lógicas de integración/desintegración de las regiones en el proceso socioeconómico. Los territorios pierden su capacidad de direccionamiento y posicionamiento en los mercados. Por ejemplo, se reduce la producción local destinada a una demanda regional, y también la capacidad de dirigir productos locales para la exportación a mercados locales de consumo tradicional.

En este contexto, podemos suponer que los territorios se ven obligados a competir de forma indiferenciada para atraer la atención de las distintas corporaciones de distribución, ya que sólo a través de su intermediación podrán integrarse en algún punto de las cadenas de valor. Progresivamente las regiones se convierten en unidades segmentarias, iguales, modulares e indiferenciadas, e incluso llegan a perder las 
ventajas (o inercias) que una tradición y una cultura productiva local -e histórica- podrían generar. Los distintos lugares llegarán -y esta es nuestra hipótesis- a insertarse en cadenas globales en la medida en que desarrollen capacidades de adaptabilidad a las condiciones productivas y, especialmente, consigan reducir la fricción territorial mediante la gestión migratoria y la movilidad de la mano de obra. Es decir, podemos considerar que la movilidad puede neutralizar las singularidades locales. La competencia puede ser activa, y ofrecer incentivos y facilidades, pero también pasiva, y permitir el proceso que acertadamente Harvey (2006) ha denominado de acumulación por desposesión. La desposesión no es sólo material, referida a la apropiación de recursos naturales o de bienes de dominio público, sino que incluye también la pérdida de derechos como pueden ser, por ejemplo, las nuevas condiciones de precarización y merma de condiciones de bienestar al amparo de la desregulación laboral.

Para explicar las transformaciones operadas en los mercados de trabajo tenemos que notar que dichos cambios se han venido produciendo de forma ligeramente distinta a las tendencias observadas en los sectores de producción industrial y de servicios. Las economías agrarias en las regiones centrales funcionaban mediante un sistema de reclutamiento laboral muy rígido, que podemos denominar como un mercado de trabajo interno, en expresión de Doeringer y Pioré (1971). Es decir, el ajuste de la mano de obra a las necesidades productivas se hacía con grandes restricciones, estaba regulado familiarmente y mediante sistemas de confianza y lealtad. Ahora, en el contexto de las migraciones transnacionales, son mercados abiertos en los que los trabajadores compiten de forma amplia en empresas, sectores y localidades. Incluso la competencia llega a desarrollarse en las propias áreas de origen en el caso de migraciones laborales. En cierta medida, la evolución ha sido inversa a la experimentada en los sectores no agrarios. En algunos subsectores de servicios, construcción o industria se han afianzado ciertos elementos de especialización basados en la experiencia laboral y en la formación que restan capacidad de movilidad intersectorial a la mano de obra. Por el contrario, en las actividades agropecuarias, la mecanización e intensificación han relajado las necesidades de conocimiento y, por ello, han contribuido al aumento de la movilidad laboral y han permitido ampliar sectorialmente su capacidad de contratación puntual.

\section{La adaptación a los regímenes agroalimentarios de la ruralidad española}

La tesis de los regímenes agroalimentarios es un marco explicativo para situar las transformaciones a nivel global. Sin embargo, esta tesis se inspira en el comportamiento de países centrales como USA, y de grandes regiones productoras como Latinoamérica mientras que la realidad de los distintos estados nación presenta particularidades (Moran et alt. 1996). Dicho esquema presenta lagunas para entender el comportamiento de áreas rurales del sur de Europa. Regiones estas que son periféricas dentro de lugares centrales, como es el caso que aquí nos ocupa. España resulta un ejemplo excepcional, el régimen autárquico que se impone desde la guerra civil hasta mediados del siglo XX la convierte, debido a su aislamiento internacional, en un observatorio particular para analizar los procesos de desagrarización sin la concomitancia de los procesos de globalización. La Guerra Civil y el triunfo del régimen dictatorial de Franco producen durante las dos décadas centrales del siglo XX un aislamiento de las corrientes del comercio internacional así como un apartamiento también de los flujos migratorios. La entrada en la ONU en 1956 simbolizará la apertura, y el Plan de Estabilización de 1959 el final del régimen autárquico. Esta larga parálisis, precisamente en el momento de fuerte mundialización económica, hace que en España podamos observar un salto abrupto desde su lugar en el primer régimen alimentario hasta su siguiente posición en el seno de las fases avanzadas del segundo régimen.

A finales del siglo XIX el peso de las economías agrarias familiares en el conjunto del sur de Europa es mayoritario. Entonces, la actividad agraria, en contextos de baja mecanización, es dependiente del trabajo y exige importantes cantidades de mano de obra para productividades reducidas. En esos años se producen 
grandes movimientos migratorios para distribuir y asignar mano de obra a los nuevos lugares de producción extensiva situados en América. Las áreas rurales de Europa funcionalmente se ocupaban de la reproducción de las sociedades locales y eran un soporte para las clases burguesas urbanas. Sin embargo, dentro del sistema-mundo realzan su función como productores de mano de obra. La perspectiva del sistema-mundo nos permite revisar la imagen de un sistema agrario cerrado y destinado al consumo interno y, en ese sentido, repensar una ruralidad falta de conexión global. La importancia del volumen que tiene la emigración, desde las áreas rurales europeas hasta las áreas rurales americanas, resulta un factor determinante para la consolidación del contemporáneo sistema agroalimentario global.

A principios del siglo XXI encontramos una configuración radicalmente distinta. Las áreas rurales han polarizado sus formas productivas. La importancia que tiene la agricultura intensiva de exportación y la importancia que tiene la inmigración como soporte laboral caracterizan una fotografía que es casi un negativo de la observada hace un siglo. En líneas generales, la posición de España en el sistema agroalimentario ha pasado de estar orientada al mercado nacional hacia una agricultura de transformación muy dirigida al mercado regional europeo y, a fecha de hoy, en apertura hacia mercados asiáticos. Estos cambios implican una transformación radical de los mercados de trabajo y de las corrientes de movilidad.

\section{Formas socioproductivas agrarias: su evolución en España}

Estas transformaciones podemos analizarlas atendiendo a las formas socioproductivas. Con formas socioproductivas nos referimos al encastre (embedment) de las formas sociales de regulación del trabajo en el sistema alimentario global. De forma sintética se expondrán cuatro momentos que serán caracterizados por la conformación del sistema de organización de la fuerza productiva y el soporte demográfico.

\section{Mercado interior con soporte de la agricultura familiar emisario de mano de obra}

Dentro del contexto del primer régimen agroalimentario, España se caracteriza por desarrollar una producción agraria que se integra en cadenas regionales de valor cuyo destino es fundamentalmente el mercado interior. El sistema agrario es un instrumento fundamental para la regulación interior; procura el mantenimiento de salarios y condiciones de vida urbanas baratas a través del control del precio de los alimentos y, sobre todo, el mantenimiento de una importante población rural como reserva de mano de obra. Buena parte de la actividad se organiza a través de grupos familiares que dedican la mayor parte del trabajo al autoconsumo. El sistema es excedentario no en producción, pero sí en mano de obra. Este excedente demográfico se trasvasa como asalariado hacia las regiones agrarias de Sudamérica y Norteamérica. Estas regiones intensifican y extienden sus capacidades productivas a través de la mano de obra procedente de países de la Europa de baja industrialización -España, Italia, Polonia-. La intensa migración es devuelta a través de la importación de materias primas. El gráfico 1 ofrece una fotografía de la fuerte dependencia de las poblaciones respecto a la producción agraria. Como se observa en dicho gráfico, en el paso del siglo XIX al XX hay un equilibrio muy inestable entre población y recursos, de forma que cualquier variación en las condiciones de producción exige una regulación migratoria. Distintas monografías han recogido estos momentos de desplazamiento de la población rural; entre ellas, se destaca por su carácter paradigmático el trabajo de Douglass y Bilbao (1975) sobre el trasvase de pastores vascos a las praderas de Nevada. El estancamiento de las economías de subsistencia, por sobredensificación, produce un excedente de mano de obra cualificada para ser empleada en condiciones de infraproletariado dentro de la emergente producción ganadera de volumen industrial en las regiones americanas. 


\section{Gráfico 1. Saldo migratorio exterior (1882-1911)}

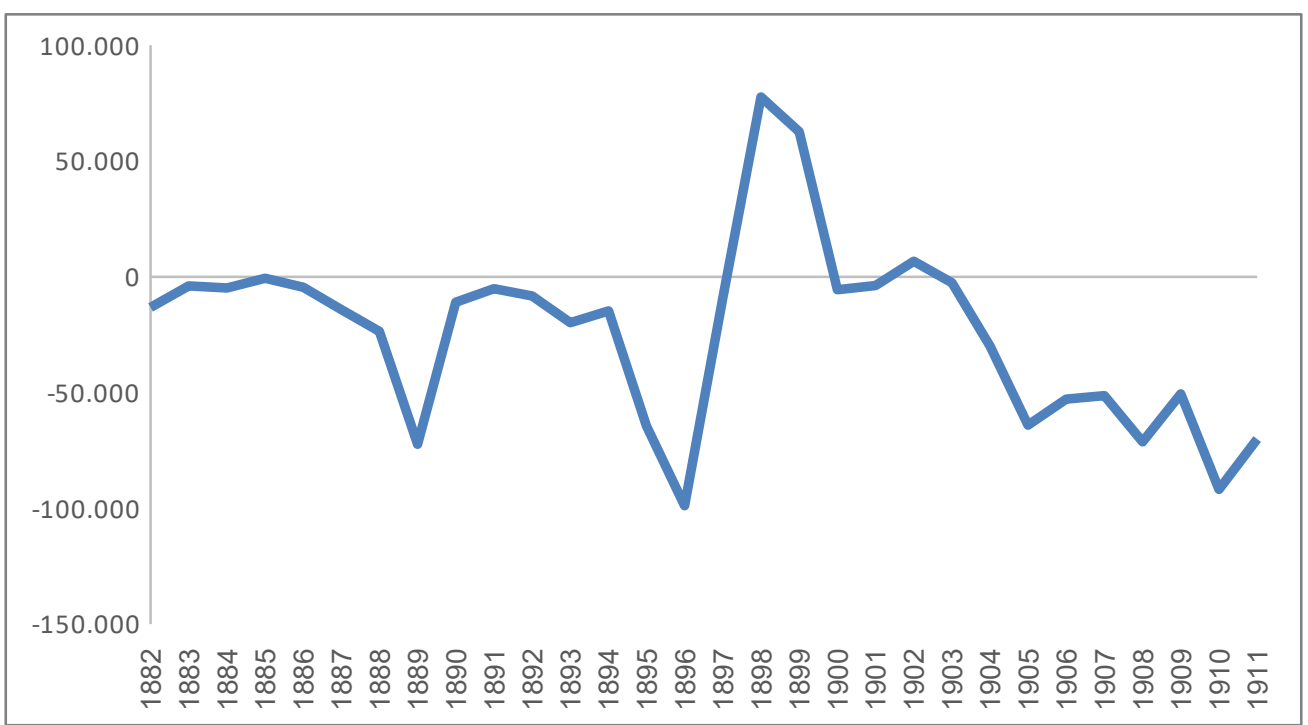

Fuente: Camarero, 1993.

\section{Salarización y desconexión}

El impacto de la Guerra Civil (1936-39) y el periodo de economía autárquica (1940-1959) aíslan a España del comercio internacional. Como consecuencia directa del desabastecimiento, el aislamiento y el deterioro de la industria se produce una reagrarización. Se invierte la tendencia a la disminución y caída de la población agraria. Sin embargo, la reagarización dará lugar a una progresiva salarización de la actividad del campo. De 1930 a 1960 la tasa de salarización agraria sube desde el 25,9 \% al 34,5 \%. Es decir, se pasa de una proporción de un asalariado de cada cuatro ocupados en la agricultura, a uno de cada tres. El hecho de que en números absolutos haya un descenso de agricultores familiares y un aumento de asalariados sugiere un importante trasvase entre ambas categorías.

\section{Tabla 1}

\begin{tabular}{|l|c|c|c|}
\hline \multicolumn{4}{|c|}{ EFECTIVOS AGRARIOS POR TIPO Y TASAS DE CRECIMIENTO 1930-1960 } \\
\hline & ASALARIADOS & $\begin{array}{c}\text { TITULARES Y } \\
\text { FAMILIARES }\end{array}$ & TOTAL \\
\hline 1930 & 1.242 & 3.560 & 4.802 \\
\hline 1960 & 1.707 & 3.240 & 4.947 \\
\hline Crecimiento 1960-1930 & 465 & -320 & 145 \\
\hline Tasa crecimiento (\%) 1960-1930 & 31,5 & $-9,4$ & 3,0 \\
\hline
\end{tabular}

Datos en miles. Fuente: Censos de Población. INE.

Estos cambios se producen en el contexto de lo que se ha denominado segunda modernización agraria (Ortí, 1997), caracterizada por el abandono del minifundismo y de la agricultura patrimonialista. Los datos señalan que se produce un abandono de las explotaciones familiares de carácter más marginal y volcadas al autoconsumo, por unas explotaciones con vocación productiva mercantil. Este proceso modernizador es inducido mediante la intervención de precios agrarios como mecanismo de regulación de los salarios 
urbanos. Se trata de un proceso de autorregulación mediante el que se liberan las explotaciones marginales y pequeñas, y se integra salarialmente a sus miembros en explotaciones mejor dimensionadas y dirigidas al mercado interior.

\section{Despoblamiento, desfamiliarización agraria e industrialización}

El final del régimen autárquico de postguerra introduce a España en el segundo régimen agroalimentario. A partir de la década de los sesenta se detecta un cambio drástico de orientación productiva: se reducen los cultivos tradicionales -trigo, viñedo y olivo-, mientras crece la ganadería estabulada y los productos de base para el consumo ganadero como la cebada (Gráfico 2). Durante estos años se produce el declive de la agricultura familiar como actividad única para el conjunto de la familia. Las unidades familiares incorporan otras ocupaciones no necesariamente agrarias. La modernización y mecanización producen un excedente poblacional de mano de obra que conforma una importante corriente de éxodo rural con destino a capitales europeas y hacia las emergentes concentraciones urbano-industriales de España. Las cifras son contundentes. Mientras que en 1950 casi la mitad de la población es rural (48 \% reside en municipios menores de 10.000 habitantes), tres décadas más tarde, en 1980, su peso se ha reducido hasta la cifra de sólo uno de cada cuatro habitantes (26,8 \%). La transferencia de mano de obra que se produce durante esos años es de tal magnitud que "la función de exportador de fuerza de trabajo ha tenido para el sector agrario durante la década de los 60 una importancia económica comparable, e incluso superior a su función tradicional de productor de mercancías”. (Leal, Leguina, Naredo, y Tarrafeta, 1986). La mecanización y modernización de estructuras agrarias además de la reducción de las economías de subsistencia producen este excedente continuo de fuerza de trabajo. En este contexto las áreas rurales priman su función como "productoras de brazos" frente a la producción de alimentos.

\section{Gráfico 2. Evolución de la superficie de los principales cultivos 1880-2000}

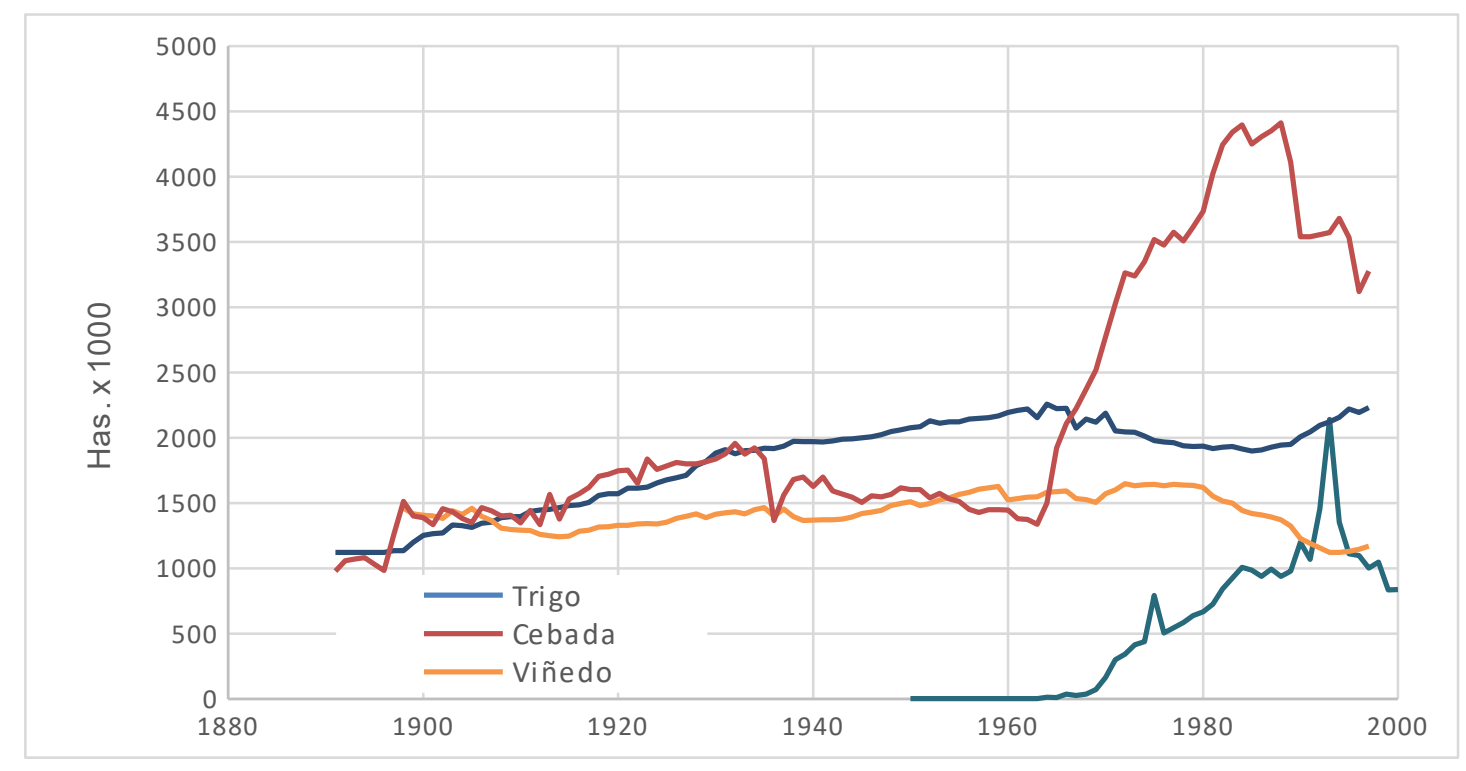

Elaboración propia a partir de datos de Carreras y Tafunell (2005).

Durante este periodo se viene observando progresivamente el descenso pronunciado de la agricultura de carácter familiar, es decir, aquélla que se realiza mediante la intervención habitual en el trabajo y la gestión productiva a través de distintos miembros de la unidad familiar (Gómez, González, y Sancho, 1999). Este proceso conocido como desfamiliarización agraria modifica y condiciona la reproducción de las poblaciones rurales y la sostenibilidad de las áreas rurales. Arnalte (1997) estimaba que para la década de los 90 aproximadamente la mitad de las explotaciones agrarias era ya de carácter individual. Estudios actuales 
(Camarero 2014) muestran que la relación entre hogares en los que sólo uno de los miembros tiene actividad agraria y hogares que pueden considerarse de agricultura familiar-aquéllos con dos o más miembros vinculados a la explotación- es de cuatro a uno a favor de los primeros ${ }^{1}$.

El proceso de desfamiliarización viene vinculado a otro proceso de corporativización familiar. Es decir, si bien desaparecen las familias que viven del trabajo de la tierra, las empresas agrarias se familiarizan. Moreno-Pérez (2012), por ejemplo, observa la importancia creciente de explotaciones multifamiliares o explotaciones que son gestionadas por miembros emparentados de varios hogares. Este proceso ha sido largamente observado en Francia. Bernard de Raymon (2013) en su análisis de la desagrarización también señala la importancia de la transición entre formas familiares y corporativas. Esta transición produce un resultado híbrido: las formas corporativas de la agricultura modernizada tienen una base familiar -en las organizaciones profesionales se nutren de grupos con relación de parentesco-, a la vez que la propia actividad agraria se desfamiliariza, y las explotaciones son de personas individuales con apoyo familiar. Arnalte (2014) hace un recorrido por la diversidad de tipos de agricultura familiar y encuentra que, paradójicamente, la pervivencia de la agricultura familiar muestra el proceso de desfamiliarización; las pequeñas unidades productivas se encuentran en retroceso, cuando no en declive, mientras las grandes explotaciones siguen en manos familiares. En lo que al proceso de desagrarización implica, esto quiere decir que la mano de obra agraria es ya casi únicamente salarial. Las familias se han reducido, y las que se han mantenido diferencian dirección y gestión de los trabajos productivos. Como resultado de este proceso se conforma el trabajo agrario mediante un mercado laboral específico y salarial del que desaparece el recurso de la mano de obra familiar.

\section{Inserción en cadenas globales y mercados competitivos}

La década de los 80 inserta la agricultura española en el mercado de la entonces Comunidad Económica Europea. Esta incorporación coincide en el tiempo con el debate sobre la emergencia y características del tercer régimen agroalimentario. Tal debate se centra en la capacidad que tiene el capital sobre las instituciones nacionales.

A finales de los años 80, las empresas y corporaciones fundamentalmente con sede en Estados Unidos presionan a Europa para que desmantele un sistema agrario fuertemente subvencionado y se liberalice de forma efectiva del mercado mundial de productos alimentarios que haga competitivas las exportaciones norteamericanas. Dentro de este contexto, se reformula la Política Agraria Común de la entonces Comunidad Económica Europea y se ponen en marcha las políticas de desarrollo rural. (Etxezarreta y Viladomiú, 1997). Con el tiempo se ha mostrado que el resultado, y probablemente el origen e intención de estas políticas, era la reconversión del sector agrario europeo. Las medidas que se plantearon con la denominación de mejoras estructurales tendieron a propiciar el establecimiento de un modelo industrial de agricultura; por ejemplo, mediante las políticas de jubilación y abandono de tierras de titulares mayores o el fomento del corporativismo a través de las Organizaciones Comunes de Mercados.

Además, estas políticas insertaron a la agricultura familiar en un modelo de complementariedad. Es decir, se potenciaba que la actividad agraria estuviera en manos de grupos industriales mientras que a las familias agrícolas se les solicitaba que redujeran su producción. De esta forma se reducían los costes de la PAC y como alternativa a la reducción de rentas a dichas familias se las implica en los procesos de desarrollo rural.

En consecuencia, se puede señalar que la modernización agraria, en cuanto proceso de descampesinización, está asociada a la progresiva desagrarización social y también a la desfamiliarización. Este proceso no es automático y produce una diversidad de situaciones. Ciertas familias se insertarán en las formas modernas de producción mercantil, pero otras quedarán atrapadas en una lógica que exige complementar los ingresos 
agrarios - de una explotación mal acogida por el mercado- con unos ingresos extraagrarios que permitan su inserción en la vida económica de la sociedad de consumo. Es decir, la agricultura se convierte en una fuente más y no necesariamente la principal para las familias rurales. El crecimiento de la pluriactividad en el contexto europeo se convierte en el síntoma de la emergencia de una agricultura crecientemente industrializada que pierde su carácter familiar y, en consonancia, su vinculación territorial.

En lo referente a la mano de obra se producen dos fenómenos de gran interés, en primer lugar, la urbanización de los activos agrarios y la extranjerización de los mismos. Fenómenos ambos, que aunque tienen causas diferentes acaban resultando complementarios para el funcionamiento de las producciones intensivas.

Los datos son claros: a finales del siglo pasado aproximadamente las dos terceras partes de los trabajadores agrarios residían en municipios de pequeño tamaño, de menos de 10.000 habitantes (Tabla 2). Tan sólo dos décadas más tarde este grupo de municipios no llega a concentrar a la mitad del conjunto de los agricultores.

Tabla 2

\begin{tabular}{|l|c|c|c|}
\hline \multicolumn{4}{|c|}{$\begin{array}{c}\text { DISTRIBUCIÓN DE LOS ACTIVOS AGRARIOS POR } \\
\text { TAMAÑO DEL MUNICIPIO DE RESIDENCIA (\%) }\end{array}$} \\
\hline & $\mathbf{1 9 9 1}$ & $\mathbf{2 0 0 1}$ & $\mathbf{2 0 1 1}$ \\
\hline Rurales & 67,3 & 55,4 & 48,5 \\
\hline Urbanos & 32,7 & 44,6 & 51,5 \\
\hline
\end{tabular}

Municipios rurales tienen una población inferior a 10.000 habitantes.

Fuente: Censos de Población. 1991, 2001 y 2011. INE. Elaboración propia.

Si bien puede parecer sorprendente la creciente importancia que adquiere el agricultor que reside en núcleos urbanos, este hecho es en cierta medida un producto de la desfamiliarización agraria que está en consonancia con el carácter restrictivo de los mercados de trabajo rurales extraagrarios. Las condiciones de los mercados de trabajo no agrarios locales son especialmente relevantes para las oportunidades laborales de las mujeres (Camarero y Sampedro, 2016). El volumen reducido, así como la poca variedad de ocupaciones amén de las pocas oportunidades formativas hacen que varios miembros de las familias que residen en el campo terminen desplazándose diariamente a centros urbanos para estudiar o para trabajar en un entorno de mayores alternativas. Pero también pueden hacerlo al revés: las familias en las que hay un agricultor pueden cambiar su residencia hacia las áreas urbanas donde encontrarán una mayor diversidad laboral y educativa para el conjunto de la familia, de forma que el miembro que desarrolla actividades agrarias pueda desplazarse diariamente hasta las fincas de trabajo. Y -como se verá a continuación- esta preferencia por la residencia urbana caracteriza también las estrategias de los asalariados agrarios. Un trabajador asalariado residente en áreas rurales está muy condicionado por la oferta estacional y tiene pocas alternativas laborales fuera de temporada. La modernización y desfamiliarización agraria han hecho también que los jornaleros pierdan el colchón de subsistencia que podía ofrecer una pequeña explotación de autoconsumo, con la que tradicionalmente los asalariados agrarios completaban sus economías. 


\section{Gráfico 3. Evolución del peso de los extranjeros por ramas de actividad}

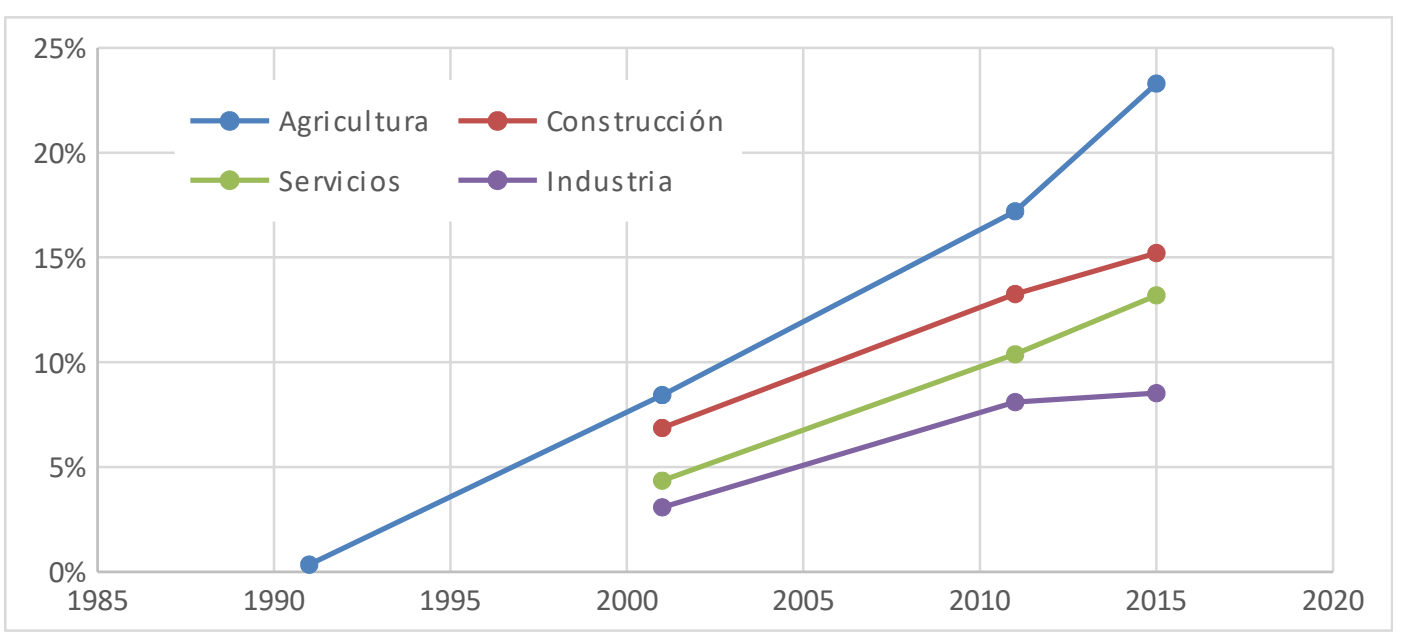

Fuente: Censos de Población 1991, 2001, 2011 y Encuesta de Población Activa 2015.

Desde principios de este siglo se incrementa significativamente la presencia de trabajadores extranjeros en las actividades agropecuarias (gráfico 3). En la actualidad suponen más de la quinta parte de los trabajadores agrarios. Según los datos de la EPA, en el segundo trimestre de 2015 el 22\% de quienes realizaron trabajos agrarios eran población extranjera. Otros trabajos como el realizado por Gadea, de Castro, Pedreño y Moraes (2015) evidencian que en áreas de agricultura intensiva y de exportación que el 50 \% de los mismos son extranjeros $\underline{2}$. Distintos estudios -Camarero, Sampedro y Oliva (2013) - han mostrado la alta rotación que tiene esta mano de obra. Los inmigrantes que llegan a zonas rurales, si bien en un primer momento pueden desarrollar trabajos agrarios, no llegan finalmente a asentarse en dichos territorios ni tampoco suelen tener continuidad en el sector agrario. Optan por la rotación entre actividades en sectores diferentes y prefieren asentarse en áreas perimetropolitanas (gráfico 4), que les permiten una accesibilidad mayor a distintas actividades laborales así como una gestión más eficaz de la estacionalidad en los sectores de agricultura, turismo, construcción o ciertos empleos gestionados a través de empresas de trabajo temporal (ETT’s).

\section{Gráfico 4. Distribución de los trabajadores agrarios por tamaño de asentamiento}

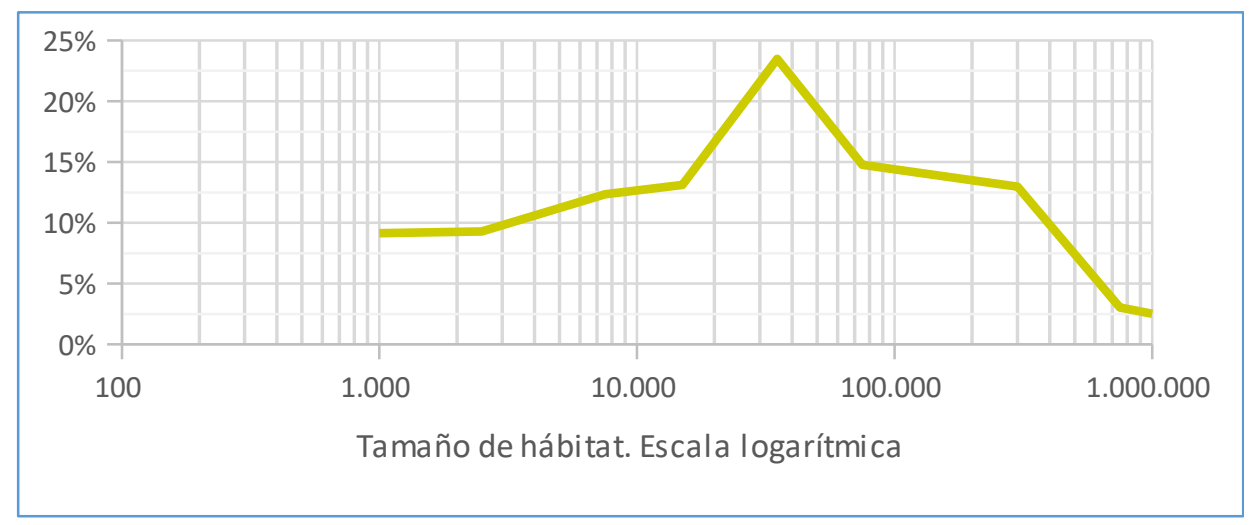

Fuente: Censo de Población. 2011. INE. Elaboración propia. 


\section{Movilidad de mano de obra y modelos socioagrarios}

La extranjerización del trabajo asalariado ha tenido como resultado el aumento de la competitividad productiva a partir de la flexibilización de la mano de obra. El efecto de la flexibilización en conjunción con la irregularidad laboral ha sido estudiado en las zonas del litoral de producción intensiva y exportadora por Pedreño (2005). Este autor destaca la importancia que tiene en las sociedades avanzadas el proceso de etnofragmentación. La diferenciación social basada en los atributos de género y etnia regula el acceso a los mercados de trabajo y en gran medida organiza el reclutamiento y acceso a los mercados de trabajo primarios y secundarios (Pioré, 1969). El género como mecanismo de segmentación en las actividades agroindustriales y en algunas tareas agrícolas ha sido ampliamente estudiado (Patel-Campillo, 2012). La generización se ha desarrollado como dispositivo de disciplinamiento de la mano de obra, de incorporación de condiciones desiguales en el trabajo y también como estrategia para el control de los flujos migratorios. En ciertos procesos de contratación en origen prima el contrato de mujeres casadas; de esta forma se garantiza la vuelta al lugar de origen una vez terminada la temporada laboral (Moreno-Nieto, 2011). Por su parte, como señala Burawoy (1976), la etnia como atributo diferenciador no sólo coloca a ciertos grupos en posición inferior en la distribución de recursos sociales y ocupacionales, sino que también permite internalizar las formas de reproducción de las unidades domésticas de las regiones de origen. La conformación de unidades residenciales diferenciales -guetto- en las principales regiones de producción agroalimentaria es una constante que ha sido documentada (Checa y Arjona, 2005). Los países centrales no importan sólo fuerza de trabajo sino también las propias condiciones de reproducción doméstica (basadas en la escasez, provisionalidad y ausencia de acumulación) de las sociedades de origen. En este sentido, la etnia se constituye en marcador, no sólo de la exclusión de actividades productivas, sino también en un sistema de segmentación social que afecta, por exclusión, a las condiciones de reproducción.

El trabajo agrario incorpora nuevas fuentes de mano de obra. Progresivamente adquieren más importancia los agricultores que por residencia son urbanos y también este ámbito se nutre cada vez más de mano de obra extranjera, multisectorial y flexible. Estas tendencias implican la reducción de dependencia respecto de los contingentes de mano de obra local que tienen las producciones territoriales. En este contexto de mercado de trabajo amplio y abierto, la actividad agraria en temporada puede competir con otros sectores de ocupación turismo, agroindustria, construcción- que son también sectores de alta rotación laboral y estacionalidad. La diferencia es que ahora los productores agrarios reducen el coste social -se reduce la demanda de alojamiento y el mantenimiento de servicios de atención fuera de temporada-.

Estas dos corrientes -urbanización y extranjerización- muestran la tendencia progresiva de deslocalización de la actividad agraria respecto al factor trabajo. Por una parte crece el número de agricultores que practican el commuting inverso y se desplazan diariamente desde residencias urbanas a campos rurales. Por otra parte crece el peso que tienen entre los asalariados los trabajadores de paso, estacionales y temporales. De esta forma se independiza a la agricultura de los mercados de trabajo locales y se trasladan las demandas de fuerza de trabajo a mercados globales. Como corolario emergen fuera de las áreas rurales nuevos enclaves que configuran territorios de asentamiento amparados en la movilidad y competencia sectorial. Ejemplo de ello es la irrupción de las ETT’s como nuevos actores de la producción agraria. (Gadea, de Castro, Pedreño y Moraes, 2015) La contratación de trabajadores se externaliza de forma que las fuentes de reclutamientos ya no son locales y los trabajadores no son necesariamente jornaleros agrarios.

En cierto sentido hay una disolución del carácter específico y particular del trabajador agrario. Ahora es un trabajador multisectorial. Esta apertura sectorial permite variar las localizaciones de la actividad agraria. La producción agraria no depende únicamente de la disponibilidad de mano de obra específica local sino que puede obtener trabajadores desde lugares periurbanos. Ciertos sectores productivos como los de productos 
perecederos de consumo fresco de la horticultura intensiva, que son procesados mediante agroindustria mecanizada asociada -el sistema que se conoce como cuarta gama- pueden acercarse a los centros de oportunidad logística -polígonos industriales y nudos de comunicación-, que concentran facilidades de transporte, de acceso a servicios y también con capacidad de recepción de mano de obra.

La actividad agraria incorpora nuevos productos y se desplaza a lugares buscando nichos de mercado concreto que producen una creciente diferenciación en el consumo. Algunas producciones ecológicas configuran nuevos paisajes productivos que aprovechan las ventajas de cercanía de consumidores y mercados a costa de convertirse en una agricultura despegada del hábitat rural.

Hay un ejemplo paradigmático que permite observar la movilidad y adaptabilidad de las producciones: el actual proceso de urbanización de las producciones ganaderas. La reciente desaparición de las cuotas lácteas $\underline{3}$ -abril de 2015- ha producido, solamente un año más tarde de su entrada en vigor, efectos evidentes en la distribución territorial de la producción. Maté (2016a, 2016b) constata una concentración de los productores en el entorno de las áreas metropolitanas, cerca de los consumidores, pero principalmente cerca de los centros de elaboración y transformación de lácteos. Las explotaciones de vacuno crecen alrededor de las grandes ciudades y puertos marítimos en detrimento de las áreas tradicionales de pastos. Un análisis de esta tendencia señala que en la producción agraria priman la reducción de costes logísticos y de transporte frente a las ventajas territoriales de producción y al uso del territorio.

Frente a estos espacios rurales dinámicos que compiten en la atracción de mano de obra, vinculados a usos no agrarios y de fuerte conexión metropolitana, encontramos territorios en declive demográfico y despoblamiento. En estos territorios la inserción en las cadenas globales de valor agrario se produce con otra lógica. Bernard de Raymon (2013) en su recorrido por la evolución de la desagrarización en Francia constata a través del análisis del boletín estadístico del Ministerio de Agricultura (Agreste 2011) la tendencia creciente hacia la especialización productiva en el sector de cereales y oleaginosas. Este proceso es paralelo al que puede observarse en las áreas del interior de España. Este autor destaca la imagen de un territorio volcado a producciones altamente mecanizadas, con apenas demanda de mano de obra, ya que por sus características pueden ser gestionadas por empresas o asociaciones profesionales. Esta imagen -campos extensivos de trabajo muy mecanizado- es también fácilmente reconocible en muchas de las zonas del interior de España y correlaciona con las regiones de fuerte vaciamiento demográfico. Este análisis resulta consistente con el panorama que en España han descrito Arnalte, Camarero y Sancho (2006) en su estudio sobre el regadío. El trabajo muestra distintas configuraciones socioproductivas dependientes de la composición demográfica y de la situación de las comarcas dentro del tejido urbano y de las oportunidades logísticas. En líneas generales, sus resultados muestran un paisaje agrario de interior volcado en cultivos extensivos de fuerte mecanización, bajo consumo de mano de obra e importante externalización a empresas de servicios agrarios. Este paisaje productivo se produce en el seno de territorios despoblados y envejecidos. Por el contrario, los espacios del litoral están volcados en la rotación de producciones de temporada con alta demanda estacional de mano de obra y productos fuertemente perecederos, baja mecanización y alta dependencia del transporte. Estos espacios están insertos en regiones de fuerte inmigración y dinamismo productivo multisectorial.

En líneas generales, podemos destacar que entre las estrategias de localización productiva, además de las propias de conexión logística, se ha buscado liberar a la actividad agraria de la presión de los mercados locales de trabajo. Las producciones de gran demanda de obra se localizan en lugares de fuerte dinamismo; prefieren competir con otros sectores a la vez que redistribuir el coste social de la reproducción de la fuerza de trabajo mediante la irregularidad generalizada y la estacionalidad intersectorial. 


\section{Nota de conclusión}

La reformulación de las actividades productivas en las regiones rurales de Europa que apuntaba la reestructuración rural puede interpretarse desde el proceso de división geográfica del trabajo (Wallerstein, 1974). La división regional entre producción y consumo es la operación central para la constitución de cadenas de valor; en función de ello, la globalización supone la diferenciación de territorios y, por ende, de sociedades locales entre sí. A su vez, también enlaza poblaciones y territorios dentro de una lógica de fuerte interdependencia. Buena parte de las áreas rurales se integran en cadenas de la industria agroalimentaria global y disuelven progresivamente su autonomía y formas organizativas propias al integrarse en relaciones y redes económicas de ámbito planetario. En el marco de los regímenes agroalimentarios (Friedman y McMichael, 1989), podemos observar como los territorios rurales son, por una parte, desposeídos de sus atributos locales en cuanto que el direccionamiento de sus capacidades productivas y de acumulación es asumido por organizaciones exógenas; por otra parte, dichos territorios tienen que desarrollar su capacidad como organización local para competir con otras organizaciones locales en su inserción en los procesos globales.

El recorrido realizado en las páginas anteriores aborda el efecto dispar que la conformación de las cadenas globales agrarias ha tenido para las áreas rurales españolas. Un efecto dispar no tanto por la diversidad de situaciones finales que pueden encontrarse, sino desigual en la medida que el análisis ha mostrado el profundo efecto de desvinculación que tienen las cadenas globales respecto de las formas socioproductivas territoriales. En líneas generales, en las áreas rurales españolas la correspondencia existente entre producciones, capacidades territoriales y mercados de trabajo se ha disuelto. Tanto las propias producciones como la mano de obra adquieren capacidad como factores independientes de movimiento, con independencia de su adecuación entre ambos elementos e incluso respecto a su relación con la vocación y tradición productiva del territorio.

Delgado (2010), entre otros, ha señalado cómo los procesos de acumulación agroalimentaria se centran en la apropiación y desposesión de lo local. Las cadenas de valor se nutren de la apropiación de recursos y del desmantelamiento de culturas productivas. La lectura realizada a través de estas páginas sugiere la desposesión de los propios territorios para ofrecer y organizar sus propios procesos productivos. En concreto, uno de los grandes efectos visibles del avance del régimen de acumulación corporativa ha sido la continua diferenciación y separación de la mano de obra del territorio. Los mecanismos han sido variados; bien mediante la puesta en marcha de masivos desplazamientos de trabajadores transcontinentales, bien por la formación de jornaleros-commuters en continua movilidad diaria para generar un colectivo flexible capaz de adaptarse a mercados de trabajo estacionales y fluctuantes en sus demandas de mano de obra.

Este proceso de movilidad de mano de obra se añade al proceso de división del trabajo que ha venido suponiendo también la movilidad de las producciones fuera de sus ámbitos tradicionales de cultivo e incluso lejos de los lugares de consumo, y en el que priman cada vez más las oportunidades de conexión y distribución.

En consecuencia, se observa que la vinculación de los territorios a las cadenas agroalimentarias no viene determinada por la oportunidad de adecuar una producción a las condiciones y cultura del propio territorio, sino por la capacidad de interiorizar y soportar costes de factores productivos, especialmente los referentes a las condiciones de vida de la mano de obra. Esta cuestión apunta a un deterioro importante de la gobernanza territorial y local de las áreas rurales.

Respecto a la cuestión que da origen a este texto el análisis realizado ha confirmado la importancia de la movilidad. Las lógicas de acumulación ganan con la movilidad. El desarrollo biotecnológico ha permitido reducir los condicionantes climáticos y edafológicos, ha permitido alargar la vida de productos perecederos hasta hacerlos acumulables o al menos transportables a cualquier lugar del planeta. Una actividad como la 
agropecuaria, que sigue necesitando al territorio en cuanto demandante de espacio, tiene en los requerimientos de las formas socioproductivas una de sus últimas fronteras. Las formas socioproductivas son formas culturales vinculadas territorialmente.

Desprenderse de un trabajador propio arraigado en el entorno y vinculado al sector es una apuesta de los nuevos enclaves productivos. En esta estrategia aparecen nuevos actores como son las ETT's que se encargan de formar bolsas de trabajo descualificado intersectoriales. Gadea, Pedreño y de Castro (2017) han mostrado la importancia que tiene la agricultura como experiencia para socializar a los nuevos trabajadores inmigrantes en condiciones de irregularidad, de forma que luego puedan, en esas mismas condiciones, ser transferidos a otros sectores -industria, construcción, turismo, etc.-- A diferencia de España, donde los enclaves productivos se localizan en áreas de fuerte commuting periurbano, en otras regiones los enclaves pueden llegar a generar nuevos asentamientos estacionales de recién llegados al área de cultivo (Lara y Sánchez, 2015), en los que son los intermediarios de la contratación-traslado quienes socializan a los trabajadores en las condiciones fordistas de producción.

Resulta paradójico que una actividad como la producción agraria, tan arraigada al terreno, haya desarrollado una necesidad tan destacable de utilizar trabajadores móviles. La paradoja muestra la importancia que tiene la desterritorialización como estrategia de acumulación. El territorio como contenedor de cultura, de formas y condiciones de regulación y de trabajo condiciona la capacidad de extracción de valor. La desvinculación de la producción agroalimentaria respecto a su origen tiene entre otras bases la movilidad de los trabajadores como requisito estratégico. La cuestión que queda por responder es qué capacidad tienen los regímenes de acumulación agroalimentaria de difundirse y contagiar a otros aspectos de la vida socioeconómica. Somos lo que comemos. Tal vez podamos añadir: "y nuestra sociedad funcionará de la misma manera en la que producimos los alimentos”.

\section{Notas}

1 En términos absolutos sólo hay 53.000 hogares que, en sentido canónico, podríamos considerar de agricultura familiar y 115.000 personas como agricultores en dicha situación.

$\underline{2}$ El estudio referido llega a mostrar cifras, en los años anteriores a la crisis, superiores al 70\%. (p. 162). Los autores utilizan datos de afiliación a la Seguridad Social Agraria

$\underline{3}$ Las cuotas lácteas eran un mecanismo de regulación del mercado de la Unión Europea. Para controlar la producción y garantizar una oferta que no sobrepasara la demanda, e implicara una caída del precio, se distribuyeron cuotas de producción entre los productores. En el caso de que algún productor sobrepasara el volumen asignado debería abonar una tasa. Este sistema sustituyó al sistema anterior de intervención de contingentes a precios regulados. Las cuotas, al repartirse territorialmente, impedían la concentración de producción y contribuían a la distribución de las explotaciones de vacuno por el conjunto de las áreas rurales.

\section{Referencias}

Agreste (2011). Les productions se concentrent dans les exploitations sepécialisées. Agreste Primeur, 272, 14.

Arnalte, E. (1997). Formas de producción y tipos de explotaciones en la agricultura española: viejas y nuevas formas de diferenciación. En C. Gómez y J.J. González, Agricultura y sociedad en la España contemporánea (pp. 501-532). Madrid: CIS-MAPA, 
Arnalte, E. (2014). Una nota sobre la agricultura familar y la naturaleza de las unidades productivas en la agricultura. Economía Agraria y Recursos Naturales, 14(1), 141-146.

Arnalte, E., Camarero. L., y Sancho, R. (2006). Los regantes. Perfiles productivos y socioprofesionales. Madrid, Ministerio de Agricultura, Pesca y Alimentación.

Bair, J. \& Werner, M. (2011). Commodity chains and the uneven geographies of global capitalism: a disarticulations perspective. Environment and Planning A, 43, 988-997.

Bernard de Raymon, A. (2013). Detaching from agriculture? Field-crop specialization as challenge to family farming in northern Côte d'Or, France. Journal of Rural Studies, 32, 283-294.

Burawoy, M. (1976). The Functions and Reproduction of Migrant Labor: Comparative Material from Southern Africa and the United States. American Journal of Sociology, 81(5), 1050-1087.

Camarero, L. (1994). Del éxodo rural y del éxodo urbano. Ocaso y renacimiento de los asentamientos rurales en España. Madrid: Ministerio de Agricultura, Pesca y Alimentación.

Camarero, L. (2014). Familias, hogares y agricultura. Agricultura Familiar en España. Anuario 2014. UPA.

Camarero, L. \& Sampedro, R. (2016). Exploring Female Over-Migration in Rural Spain- Employment, Care Giving and Mobility. In K. Wiest (Ed.), Women and Migration in Rural Europe (pp. 189-208). Nueva York: Palgrave-Macmillan.

Camarero, L. Sampedro, R., y Oliva, J. (2013). Trayectorias ocupacionales y residenciales de los inmigrantes extranjeros en las áreas rurales españolas. Sociología del Trabajo, 77, 69-91.

Carreras, A., y Tafunell, X. (Coords.). (2005). Estadísticas históricas de España: siglos XIX-XX. Bilbao: Fundación BBVA.

Castells, M. (1997) La era de la información. Economía, Sociedad y Cultura. Madrid: Alianza Editorial.

Checa, J. C., y Arjona, A. (2005). El vecino no deseado. Situación residencial de los inmigrantes africanos en Almería (España). Revue Européenne des Migrations Internationales, 21(3), 179-207.

Delgado, M. (2010). El sistema agroalimentario globalizado: Imperios alimentarios y degradación social y ecológica. Revista de Economía Crítica, 10, 32-61.

Delgado, M., Carpintero, O., Lomas, P., y Sastre, S. (2014). Andalucía en la división territorial del trabajo dentro de la economía española. Una aproximación a la luz de su metabolismo socioeconómico. 1996-2010. Revista de Estudios Regionales, 100, 197-222.

Doeringer, P. \& Pioré, M. (1971). Internal Labor Markets and Manpower Analysis. Lexington: D.C. Heat.

Douglass, W. A., \& Bilbao, J. (1975). Amerikanuak: Basques in the New World. Reno: University of Nevada Press.

Etxezarreta, M. (Ed.) (2006). La agricultura Española en la era de la globalización. Madrid: Ministerio de Agricultura, Pesca y Alimentación.

Etxezarreta, M., y Viladomiú, L. (1997). El avance hacia la internacionalización: Crónica de una década de la agricultura española. B. Gómez, y J. J. García (eds.) Agricultura y Sociedad en la España Contemporánea (pp. 317-354). Madrid: CIS-MAPA.

Friedman, H. and McMichael, P. (1989). Agriculture and the state system. The rise and decline of national agricultures, 1870 to the present. Sociologia Ruralis, 29(2), 93-117. 
Gadea, E., Pedreño, A., \& De Castro, C. (2017). Producing and mobilizing vulnerable workers: the agribussines of the region of Murcia (Spain). En A. Corrado, C. de Castro, \& D. Perrotta (Eds.), Migration and Agriculture: mobility and change in the Mediterranean area. (pp. 79-94). Londres, Routledge.

Gadea, E., de Castro, C., Pedreño, A. y Moraes, N. (2015). Jornaleros inmigrantes en la agricultura murciana: reflexiones sobre la crisis, inmigración y empleo agrícola. Migraciones, 37, 149-169.

Gómez, C., González, J. J., y Sancho, R. (1999). Identidad y profesión en la agricultura familiar española. Madrid: Centro de Investigaciones Sociológicas.

Gómez, C.; González, J. y Sancho, R. (1999). Identidad y profesión en la agricultura familiar. Madrid: Centro de Investigaciones Sociológicas.

Harvey, D. (2006). Spaces of Global Capitalism: Towards a Theory of Uneven Geographical Development. Londres: Verso.

Kayser, B. (1990). La renaissance rurale. Sociologie des campagnes du monde occidental. París: Armand Colin.

Lara, S., y Sánchez, K. (2015). En búsqueda del control. Enganche e industria de la migración en una zona productora de uva de mesa en México. En A. Riella y P. Masceroni (Comp.), Asalariados Rurales en América Latina (pp. 73-94). Montevideo: CLACSO.

Lash, S., \& Urry, J. (1987). The End of Organized Capitalism. Cambridge: Polity Press.

Lash, S., \& Urry, J. (1994). Economies of Signs \& Space. Londres, Sage.

Leal, J. L., Leguina, J., Naredo, J. M., y Tarrafeta, J. M. (1986). La agricultura en el desarrollo capitalista español. 1940-1970. 3th edn. Madrid: Siglo XXI.

Levidow, L. (2015). European transitions towards a corporate-environmental food regime: Agroecological incorporation or contestation? Journal of Rural Studies, 40, 76-89.

Lichter, D., Brown, D. L. (2011). Rural America in an Urban Society: Changing Spatial and Social Boundaries. Annual Review of Sociology. 37, 565-592.

Lowe, Ph., Marsden, T. \& Munton, R. (1990). The Social and Economic Reestructuring of Rural Britain: a position statement. Londres, ESRC Countryside Change Working Papers Series, 2, U.C.L.

Massey, D. (1984). Spatial Divisions of Labour. Social Structures and the Geography of Production. London: MacMillan.

Maté, V. (2016a). La supresión de cuotas lácteas deslocaliza la producción. El País. 7 de Febrero 2016.

Maté, V. (2016b). Más vacas cerca de las ciudades y una industria láctea más poderosa. El País. 24 de Marzo 2016.

Mather, A. S., Hill, G., \& Nijnik, M. (2006). Post-productivism and rural land use: cul de sac or challenge for theorization? Journal of Rural Studies, 22, 441-455.

McMichael, P. (2005). Global Development and the Corporate Food Regime. Research in Rural Sociology and Development, 11, 269-303.

Moran, W., Blunden, G., Workman, M., \& Bradly, A. (1996). Family Farmers, Real Regulation, and Experience of Food Regimes. Journal of Rural Studies, 12(3), 245-258. 
Moreno-Nieto, J. (2012). Movilidad transnacional, trabajo y género: temporeras marroquíes en la agricultura onubense. Política y Sociedad, 49(1), 123-140.

Moreno-Pérez, O. (2012). Revisando las categorías de análisis de la agricultura familiar: un caso de estudio del Campo de Cartagena. Revista Española de Estudios Agrosociales y Pesqueros, 232, 101-129.

Ortí, A. (1997). Una vision histórica generalista de la sociología agraria en España: las tres modernizaciones del desarrollo capitalista. En B. Gómez, y J. J. García (eds.), Agricultura y Sociedad en la España Contemporánea (pp. 71-105). Madrid: CIS-MAPA.

Patel-Campillo, A. (2012). The Gendered Production-Consumption Relation: Accounting for Emplyment and Socieconomic Hierarchies in the Colombian Cut Flower Global Commodity Chain. Sociologia Ruralis, 52(3), 272-293.

Pechlaner, G., \& Otero, G. (2008). The Third Food Regime: Neoliberal Globalism and Agricultural Biotechnology in North America. Sociologia Ruralis, 48(4), 351-371.

Pedreño, A. (2005). Sociedades etnofragmentadas. En A. Pedreño, y M. Hernández (eds.), La condición inmigrante: exploraciones e investigaciones desde la región de Murcia. Murcia: Universidad de Murcia.

Pilcher, J. M. (2006). Food in world history. Nueva York: Routledge.

Pioré, M. J. (1969). On-the-job training in dual labor markets. En A. Weber; F. Cassell y G. Woodrow (eds.), Public-private Manpower Policies (pp. 101-132). Madison: Industrial Relations Research Association,

Pistorious, R. \& van Wijk, J. (1999). The Exploitation of Plant Genetic Resources. Political Strategies in Crop Development. Wallingford: CABI Publishing.

Van der Ploeg, J. D. (2016) Theorizing Agri-Food Economies. Agriculture, 6(30). doi:10.3390/agriculture6030030

Wallerstein, I. (1974). The modern world-system. Capitalist agriculture and the origins of the European world-economy in the sixteenth century. New York: Academic Press, Inc.

Veltz, P. (1996). Mondialisation, villes et territories. L'économie d'archipel. París: Presses Universitaires de France.

Wilson, G. A. (2001). From productivism to post-productivism... and back again? Exploring the (un)changed natural and mental of European agriculture. Transactions of the Institute of British Geographers, 26, 77-102. 